\title{
Numerical Solutions of the Second-Order One-Dimensional Telegraph Equation Based on Reproducing Kernel Hilbert Space Method
}

\author{
Mustafa Inc, ${ }^{1}$ Ali Akgül, ${ }^{2,3}$ and Adem Kılıçman ${ }^{4}$ \\ ${ }^{1}$ Department of Mathematics, Science Faculty, Firat University, 23119 Elazı̆̆, Turkey \\ ${ }^{2}$ Department of Mathematics, Education Faculty, Dicle University, 21280 Diyarbakır, Turkey \\ ${ }^{3}$ Department of Mathematics and Statistics, Missouri University of Science and Technology, Rolla, MO 65409-0020, USA \\ ${ }^{4}$ Department of Mathematics and Institute for Mathematical Research, Universiti Putra Malaysia, \\ 43400 Serdang, Selangor, Malaysia
}

Correspondence should be addressed to Adem Kılıçman; kilicman@yahoo.com

Received 18 December 2012; Accepted 5 March 2013

Academic Editor: Mustafa Bayram

Copyright (C) 2013 Mustafa Inc et al. This is an open access article distributed under the Creative Commons Attribution License, which permits unrestricted use, distribution, and reproduction in any medium, provided the original work is properly cited.

We investigate the effectiveness of reproducing kernel method (RKM) in solving partial differential equations. We propose a reproducing kernel method for solving the telegraph equation with initial and boundary conditions based on reproducing kernel theory. Its exact solution is represented in the form of a series in reproducing kernel Hilbert space. Some numerical examples are given in order to demonstrate the accuracy of this method. The results obtained from this method are compared with the exact solutions and other methods. Results of numerical examples show that this method is simple, effective, and easy to use.

\section{Introduction}

The hyperbolic partial differential equations model the vibrations of structures (e.g., buildings, beams, and machines). These equations are the basis for fundamental equations of atomic physics. In this paper, we consider the telegraph equation of the form

$$
\begin{aligned}
& \frac{\partial^{2} u}{\partial t^{2}}(x, t)+2 \alpha \frac{\partial u}{\partial t}(x, t)+\beta^{2} u(x, t) \\
& =\frac{\partial^{2} u}{\partial x^{2}}(x, t)+f(x, t), \quad 0 \leq x, t \leq 1, \alpha>\beta \geq 0,
\end{aligned}
$$

with initial conditions

$$
u(x, 0)=\varphi_{1}(x), \quad u_{t}(x, 0)=\varphi_{2}(x),
$$

and appropriate boundary conditions

$$
u(0, t)=g_{0}(t), \quad u(1, t)=g_{1}(t), \quad t \geq 0
$$

by using reproducing kernel method (RKM). In recent years, much attention has been given in the literature to the development, analysis, and implementation of stable methods for the numerical solution of (1)-(3) [1-3]. Mohanty carried out a new technique to solve the linear one-spacedimensional hyperbolic equation (1) [4]. High-order accurate method for solving linear hyperbolic equation is presented in [5]. A compact finite difference approximation of fourth order for discretizing spatial derivative of linear hyperbolic equation and a collocation method for the time component are used in [6]. A numerical scheme is developed to solve the one-dimensional hyperbolic telegraph equation using the collocation points and approximating the solution using thin plate splines radial basis function [7]. Several test problems were given, and the results of numerical experiments were compared with analytical solutions to confirm the good accuracy of their scheme. Yao [8] investigated a nonlinear hyperbolic telegraph equation with an integral condition by reproducing kernel space at $\alpha=\beta=0$. Yousefi presented a numerical method for solving the one-dimensional hyperbolic telegraph equation by using Legendre multiwavelet Galerkin method [9]. Dehghan and Lakestani presented a numerical technique for the solution of the second-order 
one-dimensional linear hyperbolic equation [10]. Lakestani and Saray used interpolating scaling functions for solving (1)(3) [11]. Dehghan provided a solution of the second-order one-dimensional hyperbolic telegraph equation by using the dual reciprocity boundary integral equation (DRBIE) method [12]. The problem has explicit solution that can be obtained by the method of separation of variables in [13].

In this paper, the problem is solved easily and elegantly by using RKM. The technique has many advantages over the classical techniques. It also avoids discretization and provides an efficient numerical solution with high accuracy, minimal calculation, and avoidance of physically unrealistic assumptions. In the next section, we will describe the procedure of this method.

The theory of reproducing kernels was used for the first time at the beginning of the 20th century by Zaremba in his work on boundary value problems for harmonic and biharmonic functions [14]. Reproducing kernel theory has important application in numerical analysis, differential equations, probability, and statistics. Recently, using the RKM, some authors discussed telegraph equation [15], Troesch's porblem [16], MHD Jeffery-Hamel flow [17], Bratu's problem [18], $\mathrm{KdV}$ equation [19], fractional differential equation [20], nonlinear oscillator with discontinuity [21], nonlinear twopoint boundary value problems [22], integral equations [23], and nonlinear partial differential equations [24].

The paper is organized as follows. Section 2 introduces several reproducing kernel spaces. The representation in $W(\Omega)$ and a linear operator are presented in Section 3. Section 4 provides the main results. The exact and approximate solutions of (1)-(3) and an iterative method are developed for the kind of problems in the reproducing kernel space. We have proved that the approximate solution converges to the exact solution uniformly. Numerical experiments are illustrated in Section 5. Some conclusions are given in Section 6.

\section{Reproducing Kernel Spaces}

In this section, some useful reproducing kernel spaces are defined.

Definition 1 (reproducing kernel function). Let $E \neq \emptyset$. A function $K: E \times E \rightarrow \mathbb{C}$ is called a reproducing kernel function of the Hilbert space $H$ if and only if

(a) $K(\cdot, t) \in H$ for all $t \in E$,

(b) $\langle\varphi, K(\cdot, t)\rangle=\varphi(t)$ for all $t \in E$ and all $\varphi \in H$.

The last condition is called "the reproducing property" as the value of the function $\varphi$ at the point $t$ is reproduced by the inner product of $\varphi$ with $K(\cdot, t)$.

Definition 2. Hilbert function space $H$ is a reproducing kernel space if and only if for any fixed $x \in X$, the linear functional $I(f)=f(x)$ is bounded [25, page 5].

Definition 3. We define the space $H_{2}^{1}[0,1]$ by

$$
H_{2}^{1}[0,1]=\left\{u \in A C[0,1]: u^{\prime} \in L^{2}[0,1]\right\} .
$$

The inner product and the norm in $H_{2}^{1}[0,1]$ are defined by

$$
\begin{gathered}
\langle u, g\rangle_{H_{2}^{1}}=u(0) g(0)+\int_{0}^{1} u^{\prime}(t) g^{\prime}(t) \mathrm{d} t, \quad u, g \in H_{2}^{1}[0,1] \\
\|u\|_{H_{2}^{1}}=\sqrt{\langle u, u\rangle_{H_{2}^{1}}}, \quad u \in H_{2}^{1}[0,1] .
\end{gathered}
$$

Lemma 4. The space $H_{2}^{1}[0,1]$ is a reproducing kernel space, and its reproducing kernel function $q_{s}$ is given by [25, page 123]

$$
q_{s}(t)= \begin{cases}1+t, & t \leq s \\ 1+s, & t>s .\end{cases}
$$

Definition 5. We define the space $F_{2}^{3}[0, T]$ by

$$
\begin{array}{r}
F_{2}^{3}[0, T]=\left\{u \in A C[0, T]: u^{\prime}, u^{\prime \prime} \in A C[0, T],\right. \\
\left.u^{(3)} \in L^{2}[0, T], u(0)=u^{\prime}(0)=0\right\} .
\end{array}
$$

The inner product and the norm in $F_{2}^{3}[0, T]$ are defined by

$$
\begin{aligned}
\langle u, g\rangle_{F_{2}^{3}}= & \sum_{i=0}^{2} u^{(i)}(0) g^{(i)}(0) \\
& +\int_{0}^{T} u^{(3)}(t) g^{(3)}(t) \mathrm{d} t, \quad u, g \in F_{2}^{3}[0, T], \\
\|u\|_{F_{2}^{3}}=\sqrt{\langle u, u\rangle_{F_{2}^{3}}} & u \in F_{2}^{3}[0, T] .
\end{aligned}
$$

Lemma 6. The space $F_{2}^{3}[0, T]$ is a reproducing kernel space, and its reproducing kernel function $r_{s}$ is given by [25, page 148]

$$
r_{s}(t)=\left\{\begin{array}{r}
\frac{1}{4} s^{2} t^{2}+\frac{1}{12} s^{2} t^{3}-\frac{1}{24} s t^{4}+\frac{1}{120} t^{5}, \\
t \leq s, \\
\frac{1}{4} s^{2} t^{2}+\frac{1}{12} s^{3} t^{2}-\frac{1}{24} t s^{4}+\frac{1}{120} s^{5}, \\
t>s .
\end{array}\right.
$$

Definition 7. We define the space $W_{2}^{3}[0,1]$ by

$$
\begin{array}{r}
W_{2}^{3}[0,1]=\left\{u \in A C[0,1]: u^{\prime}, u^{\prime \prime} \in A C[0,1],\right. \\
\left.u^{(3)} \in L^{2}[0,1], u(0)=u(1)=0\right\} .
\end{array}
$$

The inner product and the norm in $W_{2}^{3}[0,1]$ are defined by

$$
\begin{aligned}
\langle u, g\rangle_{W_{2}^{3}} & =\sum_{i=0}^{2} u^{(i)}(0) g^{(i)}(0) \\
& \quad+\int_{0}^{1} u^{(3)}(x) g^{(3)}(x) \mathrm{d} x, \quad u, g \in W_{2}^{3}[0,1] \\
& \|u\|_{W_{2}^{3}}=\sqrt{\langle u, u\rangle_{W_{2}^{3}}}, \quad u \in W_{2}^{3}[0,1] .
\end{aligned}
$$

The space $W_{2}^{3}[0,1]$ is a reproducing kernel space, and its reproducing kernel function $R_{y}$ is given by the following theorem. 
Theorem 8. The space $W_{2}^{3}[0,1]$ is a reproducing kernel space, and its reproducing kernel function $R_{y}$ is given by

$$
R_{y}(x)= \begin{cases}\sum_{i=1}^{5} c_{i}(y) x^{i}, & x \leq y, \\ \sum_{i=0}^{5} d_{i}(y) x^{i}, & x>y\end{cases}
$$

where

$$
\begin{gathered}
c_{1}(y)=\frac{5}{156} y^{4}-\frac{1}{156} y^{5}-\frac{5}{26} y^{2}-\frac{5}{78} y^{3}+\frac{3}{13} y, \\
c_{2}(y)=\frac{5}{624} y^{4}-\frac{1}{624} y^{5}+\frac{21}{104} y^{2}-\frac{5}{312} y^{3}-\frac{5}{26} y, \\
c_{3}(y)=\frac{5}{1872} y^{4}-\frac{1}{1872} y^{5}+\frac{7}{104} y^{2}-\frac{5}{936} y^{3}-\frac{5}{78} y, \\
c_{4}(y)=-\frac{5}{3744} y^{4}+\frac{1}{3744} y^{5}+\frac{5}{624} y^{2}+\frac{5}{1872} y^{3}-\frac{1}{104} y, \\
c_{5}(y)=\frac{5}{3744} y^{4}-\frac{1}{18720} y^{5}-\frac{1}{624} y^{2} \\
\quad-\frac{1}{1872} y^{3}-\frac{1}{156} y+\frac{1}{120}, \\
d_{1}(y)=-\frac{1}{104} y^{4}-\frac{1}{156} y^{5}-\frac{5}{26} y^{2}-\frac{5}{78} y^{3}+\frac{3}{13} y, \\
d_{2}(y)=\frac{7}{104} y^{3}+\frac{5}{624} y^{4}-\frac{1}{624} y^{5}+\frac{21}{104} y^{2}-\frac{5}{26} y, \\
d_{3}(y)=\frac{5}{1872} y^{4}-\frac{1}{1872} y^{5}-\frac{5}{312} y^{2}-\frac{5}{936} y^{3}-\frac{5}{78} y, \\
d_{4}(y)=-\frac{5}{3744} y^{4}+\frac{1}{3744} y^{5}+\frac{5}{624} y^{2}+\frac{5}{1872} y^{3}+\frac{5}{156} y, \\
d_{5}(y)=\frac{1}{3744} y^{4}-\frac{1}{18720} y^{5}-\frac{1}{624} y^{2}-\frac{1}{1872} y^{3}-\frac{1}{156} y .
\end{gathered}
$$

Proof. Let $u \in W_{2}^{3}[0,1]$ and let $0 \leq y \leq 1$. By Definition 7 and integrating by parts two times, we obtain that

$$
\begin{aligned}
\left\langle u, R_{y}\right\rangle_{W_{2}^{3}}= & \sum_{i=0}^{2} u^{(i)}(0) R_{y}^{(i)}(0) \\
& +\int_{0}^{1} u^{(3)}(x) R_{y}^{(3)}(x) \mathrm{d} x \\
= & u(0) R_{y}(0)+u^{\prime}(0) R_{y}^{\prime}(0) \\
& +u^{\prime \prime}(0) R_{y}^{\prime \prime}(0)+u^{\prime \prime}(1) R_{y}^{(3)}(1) \\
& -u^{\prime \prime}(0) R_{y}^{(3)}(0)
\end{aligned}
$$

$$
\begin{aligned}
& -u^{\prime}(1) R_{y}^{(4)}(1)+u^{\prime}(0) R_{y}^{(4)}(0) \\
& +\int_{0}^{1} u^{\prime}(x) R_{y}^{(5)}(x) \mathrm{d} x
\end{aligned}
$$

After substituting the values of $R_{y}(0), R_{y}^{\prime}(0), R_{y}^{\prime \prime}(0), R_{y}^{(3)}(0)$, $R_{y}^{(4)}(0), R_{y}^{(3)}(1)$, and $R_{y}^{(4)}(1)$ into the above equation, we get

$$
\begin{aligned}
\left\langle u, R_{y}\right\rangle_{W_{2}^{3}} \\
=u(0) 0+u^{\prime}(0) \\
\quad \times\left(\frac{3}{13} y-\frac{5}{78} y^{3}-\frac{5}{26} y^{2}+\frac{5}{156} y^{4}-\frac{1}{156} y^{5}\right) \\
+u^{\prime \prime}(0)\left(\frac{-5}{13} y-\frac{5}{156} y^{3}+\frac{21}{52} y^{2}+\frac{5}{312} y^{4}-\frac{1}{312} y^{5}\right) \\
+u^{\prime \prime}(1) 0-u^{\prime \prime}(0) \\
\quad \times\left(\frac{-5}{13} y-\frac{5}{156} y^{3}+\frac{21}{52} y^{2}+\frac{5}{312} y^{4}-\frac{1}{312} y^{5}\right) \\
-u^{\prime}(1) 0+u^{\prime}(0) \\
\quad \times\left(-\frac{3}{13} y+\frac{5}{78} y^{3}+\frac{5}{26} y^{2}-\frac{5}{156} y^{4}+\frac{1}{156} y^{5}\right) \\
+\int_{0}^{1} u^{\prime}(x) R_{y}^{(5)}(x) \mathrm{d} x ;
\end{aligned}
$$

thus we obtain that

$$
\begin{aligned}
\left\langle u, R_{y}\right\rangle_{W_{2}^{3}} & \int_{0}^{1} u^{\prime}(x) R_{y}^{(5)}(x) \mathrm{d} x \\
= & \int_{0}^{y} u^{\prime}(x) R_{y}^{(5)}(x) \mathrm{d} x+\int_{y}^{1} u^{\prime}(x) R_{y}^{(5)}(x) \mathrm{d} x \\
= & \int_{0}^{y} u^{\prime}(x)\left(1-\frac{10}{13} y-\frac{5}{78} y^{3}-\frac{5}{26} y^{2}\right. \\
& \left.+\frac{5}{156} y^{4}-\frac{1}{156} y^{5}\right) \mathrm{d} x \\
& +\int_{y}^{1} u^{\prime}(x)\left(-\frac{10}{13} y-\frac{5}{78} y^{3}-\frac{5}{26} y^{2}\right. \\
= & (u(y)-u(0)) \\
& \times\left(1-\frac{10}{13} y-\frac{5}{78} y^{3}-\frac{5}{26} y^{2} \mathrm{~d} x\right. \\
& \left.+\frac{5}{156} y^{4}-\frac{1}{156} y^{5}\right)
\end{aligned}
$$




$$
\begin{gathered}
+(u(1)-u(y)) \\
\times\left(-\frac{10}{13} y-\frac{5}{78} y^{3}-\frac{5}{26} y^{2}\right. \\
\left.+\frac{5}{156} y^{4}-\frac{1}{156} y^{5}\right) \\
=u(y)-u(0) \\
\times\left(1-\frac{10}{13} y-\frac{5}{78} y^{3}-\frac{5}{26} y^{2}\right. \\
\left.+\frac{5}{156} y^{4}-\frac{1}{156} y^{5}\right) \\
+u(1)\left(-\frac{10}{13} y-\frac{5}{78} y^{3}-\frac{5}{26} y^{2}\right. \\
\left.+\frac{5}{156} y^{4}-\frac{1}{156} y^{5}\right) .
\end{gathered}
$$

By Definition 7, we have $u(0)=u(1)=0$. So

$$
\left\langle u, R_{y}\right\rangle_{W_{2}^{3}}=u(y) \text {. }
$$

This completes the proof.

Definition 9. We define the binary space $W(\Omega)$ by

$$
\begin{gathered}
W(\Omega)=\left\{u: \frac{\partial^{4} u}{\partial x^{2} \partial t^{2}}\right. \text { is completely continuous in } \\
\Omega=[0,1] \times[0,1], \frac{\partial^{6} u}{\partial x^{3} \partial t^{3}} \in L^{2}(\Omega), \\
u(x, 0)=0, \frac{\partial u(x, 0)}{\partial t}=0, \\
u(0, t)=0, u(1, t)=0\} .
\end{gathered}
$$

The inner product and the norm in $W(\Omega)$ are defined by

$$
\begin{aligned}
\langle u, g\rangle_{W}=\sum_{i=0}^{2} \int_{0}^{1}\left[\frac{\partial^{3}}{\partial t^{3}} \frac{\partial^{i}}{\partial x^{i}} u(0, t)\right. & \left.\times \frac{\partial^{3}}{\partial t^{3}} \frac{\partial^{i}}{\partial x^{i}} g(0, t)\right] \mathrm{d} t \\
+ & \sum_{j=0}^{2}\left\langle\frac{\partial^{j}}{\partial t^{j}} u(x, 0), \frac{\partial^{j}}{\partial t^{j}} g(x, 0)\right\rangle_{W_{2}^{3}} \\
+ & \iint_{0}^{1}\left[\frac{\partial^{3}}{\partial x^{3}} \frac{\partial^{3}}{\partial t^{3}} u(x, t)\right. \\
& \left.\times \frac{\partial^{3}}{\partial x^{3}} \frac{\partial^{3}}{\partial t^{3}} g(x, t)\right] \mathrm{d} t \mathrm{~d} x, \\
\|u\|_{W}= & \sqrt{\langle u, u\rangle_{W}}, \quad u \in W(\Omega) .
\end{aligned}
$$

Lemma 10. $W(\Omega)$ is a reproducing kernel space, and its reproducing kernel function $K_{(y, s)}$ is given by [25, page 148]

$$
K_{(y, s)}=R_{y} r_{s}
$$

Definition 11 . We define the binary space $\widehat{W}(\Omega)$ by

$$
\begin{gathered}
\widehat{W}(\Omega)=\{u: u \text { is completely continuous in } \\
\left.\Omega=[0,1] \times[0,1], \frac{\partial^{2} u}{\partial x \partial t} \in L^{2}(\Omega)\right\} .
\end{gathered}
$$

The inner product and the norm in $\widehat{W}(\Omega)$ are defined by

$$
\begin{aligned}
\langle u, g\rangle_{\widehat{W}}= & \int_{0}^{1}\left[\frac{\partial}{\partial t} u(0, t) \frac{\partial}{\partial t} g(0, t)\right] \mathrm{d} t \\
& +\langle u(x, 0), g(x, 0)\rangle_{H_{2}^{1}} \\
& +\iint_{0}^{1}\left[\frac{\partial}{\partial x} \frac{\partial}{\partial t} u(x, t) \frac{\partial}{\partial x} \frac{\partial}{\partial t} g(x, t)\right] \mathrm{d} t \mathrm{~d} x, \\
\|u\|_{\widehat{W}} & =\sqrt{\langle u, u\rangle_{\widehat{W}}}, \quad u \in \widehat{W}(\Omega) .
\end{aligned}
$$

Lemma 12. $\widehat{W}(\Omega)$ is a reproducing kernel space, and its reproducing kernel function $G_{(y, s)}$ is given as [25, page 148]

$$
G_{(y, s)}=Q_{y} q_{s}
$$

Remark 13. Hilbert spaces can be completely classified: there is a unique Hilbert space up to isomorphism for every cardinality of the base. Since finite-dimensional Hilbert spaces are fully understood in linear algebra and since morphisms of Hilbert spaces can always be divided into morphisms of spaces with Aleph-null $\left(\chi_{0}\right)$ dimensionality, functional analysis of Hilbert spaces mostly deals with the unique Hilbert space of dimensionality Aleph-null and its morphisms. One of the open problems in functional analysis is to prove that every bounded linear operator on a Hilbert space has a proper invariant subspace. Many special cases of this invariant subspace problem have already been proven [26].

\section{Solution Representation in $W(\Omega)$}

In this section, the solution of (1) is given in the reproducing kernel space $W(\Omega)$. On defining the linear operator $L: W(\Omega)$ $\rightarrow \widehat{W}(\Omega)$ by

$$
L v=\frac{\partial^{2} v}{\partial t^{2}}-\frac{\partial^{2} v}{\partial x^{2}}+2 \alpha \frac{\partial v}{\partial t}+\beta^{2} v(x, t),
$$

after homogenizing the initial and boundary conditions, model problem (1)-(3) changes to the problem

$$
\begin{gathered}
L v=M(x, t), \quad(x, t) \in[0,1] \times[0,1], \\
v(x, 0)=\frac{\partial v}{\partial t}(x, 0)=v(0, t)=v(1, t)=0,
\end{gathered}
$$


where

$$
\begin{aligned}
M(x, t)= & \frac{\partial^{2} Z}{\partial t^{2}}(x, t)-\frac{\partial^{2} v}{\partial x^{2}}(x, t) \\
& +2 \alpha \frac{\partial Z}{\partial t}(x, t)+\beta^{2} Z(x, t)+f(x, t) ;
\end{aligned}
$$

for convenience, we again write $u$ instead of $v$ in (26)

Lemma 14. $L$ is a bounded linear operator.

Proof. Let $u \in W(\Omega)$ and let $(x, t) \in \Omega$. By Lemma 10, we have

$$
u(x, t)=\left\langle u, K_{(x, t)}\right\rangle_{W}
$$

and thus

$$
\begin{aligned}
L u(x, t) & =\left\langle u, L K_{(x, t)}\right\rangle_{W}, \\
\frac{\partial}{\partial x} L u(x, t) & =\left\langle u, \frac{\partial}{\partial x} L K_{(x, t)}\right\rangle_{W}, \\
\frac{\partial}{\partial t} L u(x, t) & =\left\langle u, \frac{\partial}{\partial t} L K_{(x, t)}\right\rangle_{W}, \\
\frac{\partial}{\partial t} \frac{\partial}{\partial x} L u(x, t) & =\left\langle u, \frac{\partial}{\partial t} \frac{\partial}{\partial x} L K_{(x, t)}\right\rangle_{W} .
\end{aligned}
$$

Hence there exist $a_{0}, b_{0}, a_{1}, b_{1}>0$ such that

$$
\begin{gathered}
|L u(x, t)| \leq a_{0}\|u\|_{W}, \\
\left|\frac{\partial}{\partial t} L u(x, t)\right| \leq b_{0}\|u\|_{W}, \\
\left|\frac{\partial}{\partial x} L u(x, t)\right| \leq a_{1}\|u\|_{W}, \\
\left|\frac{\partial}{\partial t} \frac{\partial}{\partial x} L u(x, t)\right| \leq b_{1}\|u\|_{W} .
\end{gathered}
$$

Therefore,

$$
\begin{aligned}
\|L u\|_{\widehat{W}}^{2}= & \int_{0}^{1}\left[\frac{\partial}{\partial t} L u(0, t)\right]^{2} \mathrm{~d} t \\
& +\langle L u(x, 0), L u(x, 0)\rangle_{H_{2}^{1}} \\
& +\iint_{0}^{1}\left[\frac{\partial}{\partial x} \frac{\partial}{\partial t} L u(x, t)\right]^{2} \mathrm{~d} t \mathrm{~d} x \\
= & \int_{0}^{1}\left[\frac{\partial}{\partial t} L u(0, t)\right]^{2} \mathrm{~d} t+[L u(0,0)]^{2} \\
& +\int_{0}^{1}\left[\frac{\partial}{\partial x} L u(x, 0)\right]^{2} \mathrm{~d} x \\
& +\iint_{0}^{1}\left[\frac{\partial}{\partial x} \frac{\partial}{\partial t} L u(x, t)\right]^{2} \mathrm{~d} t \mathrm{~d} x \\
\leq & \left(a_{0}^{2}+a_{1}^{2}+b_{0}^{2}+b_{1}^{2}\right)\|u\|_{W}^{2} .
\end{aligned}
$$

This completes the proof.
Now, choose a countable dense subset $\left\{\left(x_{1}, t_{1}\right),\left(x_{2}\right.\right.$, $\left.\left.t_{2}\right), \ldots\right\}$ in $\Omega$ and define

$$
\varphi_{i}=G_{\left(x_{i}, t_{i}\right)}, \quad \Psi_{i}=L^{*} \varphi_{i}
$$

where $L^{*}$ is the adjoint operator of $L$. The orthonormal system $\left\{\widehat{\Psi}_{i}\right\}_{i=1}^{\infty}$ of $W(\Omega)$ can be derived from the process of GramSchmidt orthogonalization of $\left\{\Psi_{i}\right\}_{i=1}^{\infty}$ as

$$
\widehat{\Psi}_{i}=\sum_{k=1}^{i} \beta_{i k} \Psi_{k} .
$$

Theorem 15. Suppose that $\left\{\left(x_{i}, t_{i}\right)\right\}_{i=1}^{\infty}$ is dense in $\Omega$. Then $\left\{\Psi_{i}\right\}_{i=1}^{\infty}$ is a complete system in $W(\Omega)$, and

$$
\Psi_{i}=L K_{\left(x_{i}, t_{i}\right)}(x, t)
$$

Proof. We have

$$
\begin{aligned}
\Psi_{i} & =L^{*} \varphi_{i}=\left\langle L^{*} \varphi_{i}, K_{(x, t)}\right\rangle_{W} \\
& =\left\langle\varphi_{i}, L K_{(x, t)}\right\rangle_{\widehat{W}} \\
& =\left\langle L K_{(x, t)}, G_{\left(x_{i}, t_{i}\right)}\right\rangle_{\widehat{W}} \\
& =L K_{(x, t)}\left(x_{i}, t_{i}\right) \\
& =L K_{\left(x_{i}, t_{i}\right)}(x, t) .
\end{aligned}
$$

Clearly, $\Psi_{i} \in W(\Omega)$. For each fixed $u \in W(\Omega)$, if

$$
\left\langle u, \Psi_{i}\right\rangle_{W}=0, \quad i=1,2, \ldots,
$$

then

$$
\begin{aligned}
0 & =\left\langle u, \Psi_{i}\right\rangle_{W} \\
& =\left\langle u, L^{*} \varphi_{i}\right\rangle_{W} \\
& =\left\langle L u, \varphi_{i}\right\rangle_{\widehat{W}} \\
& =L u\left(x_{i}, t_{i}\right), \quad i=1,2, \ldots
\end{aligned}
$$

Note that $\left\{\left(x_{i}, t_{i}\right)\right\}_{i=1}^{\infty}$ is dense in $\Omega$. Hence, $L u=0$. From the existence of $L^{-1}$, it follows that $u=0$. The proof is completed.

Theorem 16. If $\left\{\left(x_{i}, t_{i}\right)\right\}_{i=1}^{\infty}$ is dense in $\Omega$, then the solution of (26) is given by

$$
u=\sum_{i=1}^{\infty} \sum_{k=1}^{i} \beta_{i k} M\left(x_{k}, t_{k}\right) \widehat{\Psi}_{i} .
$$


Proof. By Theorem 15, $\left\{\Psi_{i}(x, t)\right\}_{i=1}^{\infty}$ is a complete system in $W(\Omega)$. Thus,

$$
\begin{aligned}
u & =\sum_{i=1}^{\infty}\left\langle u, \widehat{\Psi}_{i}\right\rangle_{W} \widehat{\Psi}_{i} \\
& =\sum_{i=1}^{\infty} \sum_{k=1}^{i} \beta_{i k}\left\langle u, \Psi_{k}\right\rangle_{W} \widehat{\Psi}_{i} \\
& =\sum_{i=1}^{\infty} \sum_{k=1}^{i} \beta_{i k}\left\langle u, L^{*} \varphi_{k}\right\rangle_{W} \widehat{\Psi}_{i} \\
& =\sum_{i=1}^{\infty} \sum_{k=1}^{i} \beta_{i k}\left\langle L u, \varphi_{k}\right\rangle_{\widehat{W}} \widehat{\Psi}_{i} \\
& =\sum_{i=1}^{\infty} \sum_{k=1}^{i} \beta_{i k}\left\langle L u, G_{\left(x_{k}, t_{k}\right)}\right\rangle_{\widehat{W}} \widehat{\Psi}_{i} \\
& =\sum_{i=1}^{\infty} \sum_{k=1}^{i} \beta_{i k} L u\left(x_{k}, t_{k}\right) \widehat{\Psi}_{i} \\
& =\sum_{i=1}^{\infty} \sum_{k=1}^{i} \beta_{i k} M\left(x_{k}, t_{k}\right) \widehat{\Psi}_{i} .
\end{aligned}
$$

This completes the proof.

Now the approximate solution $u_{n}$ can be obtained from the $n$-term intercept of the exact solution $u$ and

$$
u_{n}=\sum_{i=1}^{n} \sum_{k=1}^{i} \beta_{i k} M\left(x_{k}, t_{k}\right) \widehat{\Psi}_{i} .
$$

Obviously

$$
\left\|u_{n}-u\right\|_{W} \longrightarrow 0, \quad n \longrightarrow \infty .
$$

Theorem 17. If $u \in W(\Omega)$, then

$$
\left\|u_{n}-u\right\|_{W} \longrightarrow 0, \quad n \longrightarrow \infty .
$$

Moreover, a sequence $\left\|u_{n}-u\right\|_{W}$ is monotonically decreasing in $n$.

Proof. From (38) and (40), it follows that

$$
\left\|u_{n}-u\right\|_{W}=\left\|\sum_{i=n+1}^{\infty} \sum_{k=1}^{i} \beta_{i k} M\left(x_{k}, t_{k}\right) \widehat{\Psi}_{i}(x, t)\right\|_{W} .
$$

Thus,

$$
\left\|u_{n}(x, t)-u(x, t)\right\|_{W} \longrightarrow 0, \quad n \longrightarrow \infty .
$$

In addition

$$
\begin{aligned}
\left\|u_{n}-u\right\|_{W}^{2} & =\left\|\sum_{i=n+1}^{\infty} \sum_{k=1}^{i} \beta_{i k} M\left(x_{k}, t_{k}\right) \widehat{\Psi}_{i}(x, t)\right\|_{W}^{2} \\
& =\sum_{i=n+1}^{\infty}\left(\sum_{k=1}^{i} \beta_{i k} M\left(x_{k}, t_{k}\right) \widehat{\Psi}_{i}(x, t)\right)^{2} .
\end{aligned}
$$

Clearly, $\left\|u_{n}(x, t)-u(x, t)\right\|_{W}$ is monotonically decreasing in $n$.

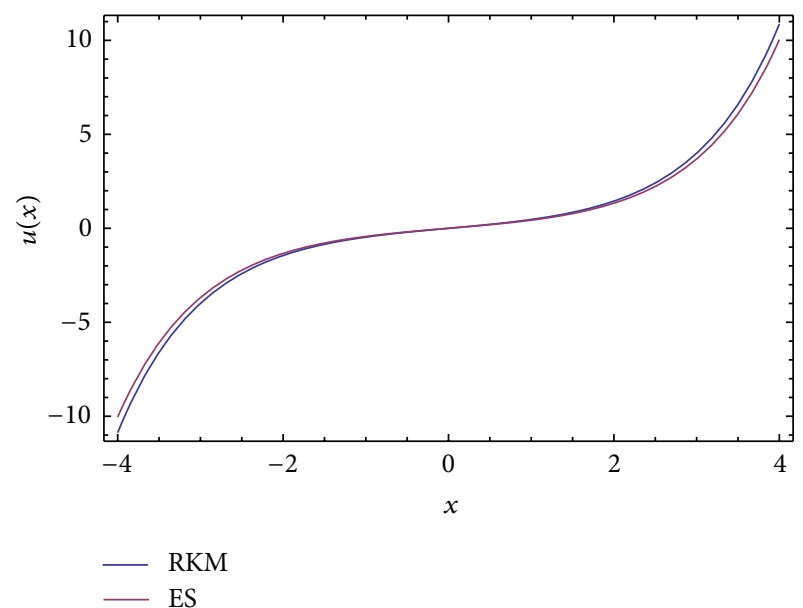

Figure 1: Graph of numerical results for Example $20(\alpha=10, \beta=$ $20, t=0.5)$.

\section{The Method Implementation}

(i) If (26) is linear, then the analytical solution of (26) can be obtained directly by (38).

(ii) If (26) is nonlinear, then the solution of (26) can be obtained by the following iterative method.

We construct an iterative sequence $u_{n}$, putting

any fixed $u_{0} \in W_{2}^{3}[0,1]$,

$$
u_{n}=\sum_{i=1}^{n} A_{i} \widehat{\Psi}_{i}
$$

where

$$
\begin{gathered}
A_{1}=\beta_{11} M\left(x_{k}, t_{k}, u\left(x_{k}, t_{k}\right)\right) \\
A_{2}=\sum_{k=1}^{2} \beta_{2 k} M\left(x_{k}, t_{k}, u_{k-1}\left(x_{k}, t_{k}\right)\right),
\end{gathered}
$$

$$
A_{n}=\sum_{k=1}^{n} \beta_{n k} M\left(x_{k}, t_{k}, u_{k-1}\left(x_{k}, t_{k}\right)\right) .
$$

Next we will prove that $u_{n}$ given by the iterative formula (46) converges to the exact solution.

Theorem 18. Suppose that $\left\|u_{n}\right\|$ defined by (46) is bounded and (26) has a unique solution. If $\left\{\left(x_{i}, t_{i}\right)\right\}_{i=1}^{\infty}$ is dense in $\Omega$, then $u_{n}$ converges to the analytical solution $u$ of (26), and

$$
u=\sum_{i=1}^{\infty} A_{i} \widehat{\Psi}_{i}
$$

where $A_{i}$ is given by (47). 
TABLE 1: Numerical results for Example 20 for $t=0.5$.

\begin{tabular}{lccccccc}
\hline & & $\mathrm{AE}$ & $\mathrm{RE}$ & $\mathrm{AE}$ & $\mathrm{RE}$ & $\mathrm{AE}$ & $\mathrm{RE}$ \\
$x$ & $\mathrm{CPU}$ time (s) & $\alpha=20$ & $\alpha=20$ & $\alpha=10$ & $\alpha=10$ & $\alpha=50$ & $\alpha=50$ \\
& & $\beta=10$ & $\beta=10$ & $\beta=5$ & $\beta=5$ & $\beta=2$ & $\beta=2$ \\
\hline 0.9829 & 2.168 & $4.9 \times 10^{-9}$ & $1.1 \times 10^{-7}$ & $8.9 \times 10^{-8}$ & $2.0 \times 10^{-6}$ & $9.6 \times 10^{-9}$ & $2.1 \times 10^{-7}$ \\
0.9903 & 2.324 & $3.8 \times 10^{-8}$ & $1.5 \times 10^{-7}$ & $4.6 \times 10^{-8}$ & $1.9 \times 10^{-7}$ & $9.8 \times 10^{-7}$ & $4.0 \times 10^{-6}$ \\
0.9938 & 2.215 & $5.9 \times 10^{-8}$ & $3.6 \times 10^{-6}$ & $5.0 \times 10^{-8}$ & $3.1 \times 10^{-6}$ & $8.5 \times 10^{-9}$ & $5.2 \times 10^{-7}$ \\
0.9957 & 2.262 & $6.8 \times 10^{-7}$ & $2.5 \times 10^{-7}$ & $1.4 \times 10^{-7}$ & $5.3 \times 10^{-7}$ & $5.2 \times 10^{-7}$ & $1.9 \times 10^{-6}$ \\
\hline
\end{tabular}

TABLE 2: Numerical results for Example 20 for $t=1.0$.

\begin{tabular}{cccccccc}
\hline & & AE & RE & AE & RE & AE & RE \\
$x$ & CPU time (s) & $\alpha=20$ & $\alpha=20$ & $\alpha=10$ & $\alpha=10$ & $\alpha=50$ & $\alpha=50$ \\
& & $\beta=10$ & $\beta=10$ & $\beta=5$ & $\beta=5$ & $\beta=2$ & $\beta=2$ \\
\hline 0.9829 & 2.340 & $5.1 \times 10^{-9}$ & $3.1 \times 10^{-7}$ & $5.5 \times 10^{-8}$ & $3.3 \times 10^{-6}$ & $4.5 \times 10^{-7}$ & $2.7 \times 10^{-5}$ \\
0.9903 & 2.278 & $7.1 \times 10^{-7}$ & $7.8 \times 10^{-6}$ & $1.6 \times 10^{-8}$ & $1.8 \times 10^{-7}$ & $9.5 \times 10^{-7}$ & $1.0 \times 10^{-5}$ \\
0.9938 & 2.293 & $4.4 \times 10^{-7}$ & $7.3 \times 10^{-5}$ & $1.7 \times 10^{-6}$ & $2.9 \times 10^{-4}$ & $2.2 \times 10^{-7}$ & $3.6 \times 10^{-5}$ \\
0.9957 & 2.277 & $2.2 \times 10^{-8}$ & $2.2 \times 10^{-7}$ & $1.4 \times 10^{-8}$ & $1.4 \times 10^{-7}$ & $7.2 \times 10^{-7}$ & $7.4 \times 10^{-6}$ \\
\hline
\end{tabular}

TABLE 3: A comparison between interpolating scaling function method [11] and RKM for different values of $\alpha, \beta$, and $t$ for Example 20.

\begin{tabular}{cccccc}
\hline & & $\mathrm{AE}$ & $\mathrm{AE}$ & $\mathrm{AE}$ & $\mathrm{AE}$ \\
$x$ & $\begin{array}{c}\mathrm{CPU} \text { time } \\
(\mathrm{s})\end{array}$ & $\begin{array}{c}\alpha=20 \\
t=0.5\end{array}$ & $\begin{array}{c}\alpha=20 \\
t=0.5\end{array}$ & $\begin{array}{c}\beta=10 \\
t=1\end{array}$ & $\begin{array}{c}\beta=10 \\
t=1\end{array}$ \\
\hline 0.0 & 2.512 & $2 \times 10^{-21}$ & 0.0 & $2 \times 10^{-6}$ & 0.0 \\
0.1 & 2.262 & $5 \times 10^{-4}$ & $3 \times 10^{-7}$ & $1 \times 10^{-3}$ & $3 \times 10^{-8}$ \\
0.2 & 2.309 & $7 \times 10^{-4}$ & $2 \times 10^{-8}$ & $2 \times 10^{-3}$ & $3 \times 10^{-7}$ \\
0.3 & 2.293 & $1 \times 10^{-3}$ & $2 \times 10^{-7}$ & $2 \times 10^{-4}$ & $2 \times 10^{-7}$ \\
0.4 & 2.278 & $2 \times 10^{-3}$ & $5 \times 10^{-5}$ & $4 \times 10^{-4}$ & $1 \times 10^{-7}$ \\
0.5 & 2.883 & $3 \times 10^{-3}$ & $1 \times 10^{-5}$ & $5 \times 10^{-4}$ & $8 \times 10^{-8}$ \\
0.6 & 2.821 & $3 \times 10^{-3}$ & $5 \times 10^{-8}$ & $8 \times 10^{-4}$ & $5 \times 10^{-7}$ \\
0.7 & 2.805 & $4 \times 10^{-3}$ & $2 \times 10^{-7}$ & $8 \times 10^{-4}$ & $1 \times 10^{-7}$ \\
0.8 & 2.231 & $3 \times 10^{-3}$ & $6 \times 10^{-7}$ & $6 \times 10^{-4}$ & $1 \times 10^{-7}$ \\
0.9 & 2.277 & $2 \times 10^{-3}$ & $2 \times 10^{-6}$ & $3 \times 10^{-4}$ & $8 \times 10^{-8}$ \\
1.0 & 2.169 & $2 \times 10^{-4}$ & $2 \times 10^{-8}$ & $9 \times 10^{-5}$ & $4 \times 10^{-10}$ \\
\hline
\end{tabular}

Proof. First, we prove the convergence of $u_{n}$. From (46) and the orthonormality of $\left\{\widehat{\Psi}_{i}\right\}_{i=1}^{\infty}$, we infer that

$$
\begin{aligned}
\left\|u_{n+1}\right\|^{2} & =\sum_{i=1}^{n+1} A_{i}^{2}=\sum_{i=1}^{n} A_{i}^{2}+A_{n+1}^{2} \\
& =\left\|u_{n}\right\|^{2}+A_{n+1}^{2} \geq\left\|u_{n}\right\| .
\end{aligned}
$$

By (49), $\left\|u_{n}\right\|$ is nondecreasing, and by the assumption, $\left\|u_{n}\right\|$ is bounded. Thus $\left\|u_{n}\right\|$ is convergent. By (49), there exists a constant $c$ such that

$$
\sum_{i=1}^{\infty} A_{i}^{2}=c .
$$

TABLE 4: A comparison between interpolating scaling function method [11] and RKM for different values of $\alpha, \beta$, and $t$ for Example 20.

\begin{tabular}{ccccc}
\hline & $\mathrm{AE}$ & $\mathrm{AE}$ & $\mathrm{AE}$ & $\mathrm{AE}$ \\
$x$ & {$[11]$} & $\mathrm{RKM}$ & {$[11]$} & $\mathrm{RKM}$ \\
& $\alpha=10$ & $\alpha=10$ & $\beta=5$ & $\beta=5$ \\
& $t=0.5$ & $t=0.5$ & $t=1$ & $t=1$ \\
\hline 0.0 & 0.0 & 0.0 & $2 \times 10^{-6}$ & 0.0 \\
0.1 & $3 \times 10^{-4}$ & $1 \times 10^{-7}$ & $1 \times 10^{-4}$ & $2 \times 10^{-7}$ \\
0.2 & $1 \times 10^{-3}$ & $3 \times 10^{-8}$ & $7 \times 10^{-4}$ & $2 \times 10^{-6}$ \\
0.3 & $1 \times 10^{-3}$ & $9 \times 10^{-8}$ & $6 \times 10^{-4}$ & $6 \times 10^{-7}$ \\
0.4 & $2 \times 10^{-3}$ & $2 \times 10^{-8}$ & $9 \times 10^{-4}$ & $7 \times 10^{-8}$ \\
0.5 & $2 \times 10^{-3}$ & $2 \times 10^{-7}$ & $1 \times 10^{-3}$ & $5 \times 10^{-8}$ \\
0.6 & $2 \times 10^{-3}$ & $5 \times 10^{-7}$ & $1 \times 10^{-3}$ & $9 \times 10^{-8}$ \\
0.7 & $2 \times 10^{-3}$ & $7 \times 10^{-8}$ & $8 \times 10^{-4}$ & $3 \times 10^{-8}$ \\
0.8 & $2 \times 10^{-3}$ & $7 \times 10^{-8}$ & $7 \times 10^{-4}$ & $6 \times 10^{-8}$ \\
0.9 & $1 \times 10^{-3}$ & $4 \times 10^{-7}$ & $3 \times 10^{-4}$ & $2 \times 10^{-8}$ \\
1.0 & $1 \times 10^{-4}$ & $8 \times 10^{-9}$ & $9 \times 10^{-5}$ & $7 \times 10^{-8}$ \\
\hline
\end{tabular}

This implies that

$$
\left\{A_{i}\right\}_{i=1}^{\infty} \in \ell^{2} .
$$

If $m>n$, then

$$
\begin{aligned}
\left\|u_{m}-u_{n}\right\|^{2} & =\left\|\sum_{k=n}^{m-1} u_{k+1}-u_{k}\right\|^{2} \\
& \leq \sum_{k=n}^{m-1}\left\|u_{k+1}-u_{k}\right\|^{2} \\
& =\sum_{k=n}^{m-1} A_{k+1}^{2} \longrightarrow 0, \quad m, n \longrightarrow \infty .
\end{aligned}
$$


TABLE 5: Numerical results for Example 21 for $t=0.5$.

\begin{tabular}{lccccccc}
\hline & & $\mathrm{AE}$ & $\mathrm{RE}$ & $\mathrm{AE}$ & $\mathrm{RE}$ & $\mathrm{AE}$ & $\mathrm{RE}$ \\
$x$ & $\mathrm{CPU}$ time (s) & $\alpha=20$ & $\alpha=20$ & $\alpha=10$ & $\alpha=10$ & $\alpha=50$ & $\beta=2$ \\
& & $\beta=10$ & $\beta=10$ & $\beta=5$ & $\beta=5$ & $\beta=2$ \\
\hline 0.9829 & 2.215 & $7.23 \times 10^{-8}$ & $6.8 \times 10^{-7}$ & $3.4 \times 10^{-9}$ & $3.2 \times 10^{-8}$ & $8.41 \times 10^{-8}$ & $7.99 \times 10^{-7}$ \\
0.9903 & 2.246 & $3.51 \times 10^{-9}$ & $6.8 \times 10^{-9}$ & $1.8 \times 10^{-9}$ & $3.5 \times 10^{-9}$ & $3.52 \times 10^{-7}$ & $6.86 \times 10^{-7}$ \\
0.9938 & 2.231 & $7.56 \times 10^{-7}$ & $1.9 \times 10^{-5}$ & $7.6 \times 10^{-8}$ & $1.9 \times 10^{-6}$ & $2.84 \times 10^{-9}$ & $7.28 \times 10^{-8}$ \\
0.9957 & 2.433 & $5.45 \times 10^{-8}$ & $9.9 \times 10^{-8}$ & $2.6 \times 10^{-8}$ & $4.8 \times 10^{-8}$ & $4.53 \times 10^{-8}$ & $8.27 \times 10^{-8}$ \\
\hline
\end{tabular}

TABLE 6: Numerical results for Example 21 for $t=1.0$.

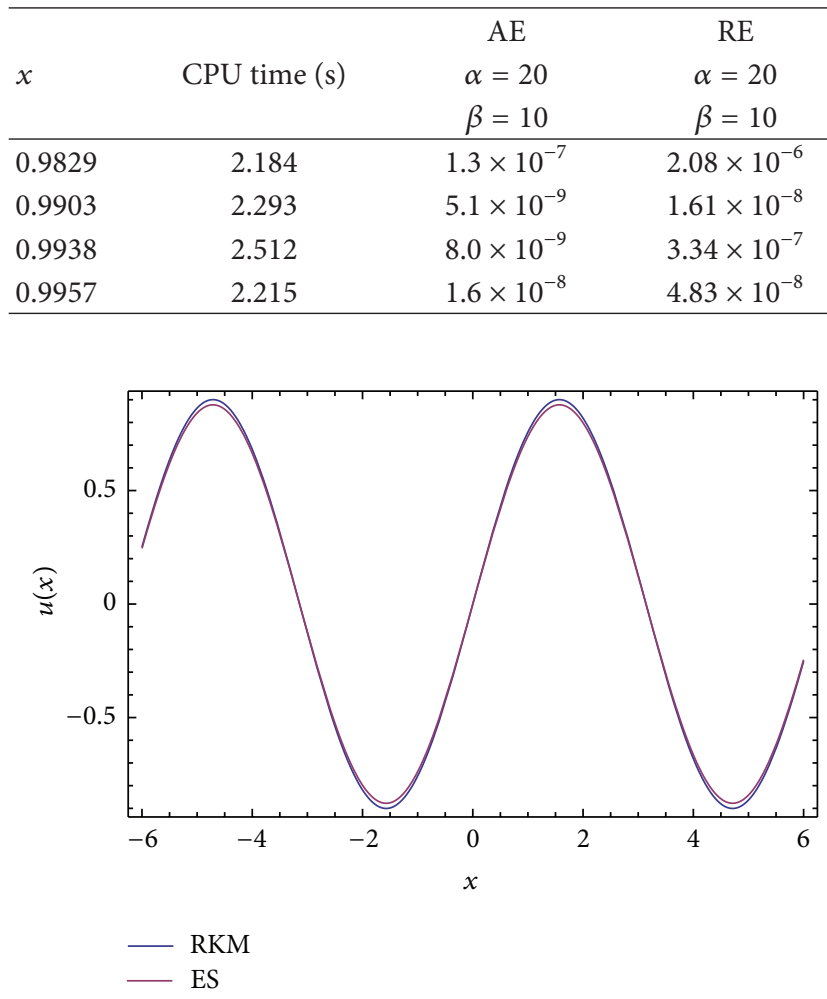

Figure 2: Graph of numerical results for Example $21(\alpha=10, \beta=$ $20, t=0.5)$.

The completeness of $W(\Omega)$ shows that there exists $\widehat{u} \in W(\Omega)$ such that $u_{n} \rightarrow \widehat{u}$ as $n \rightarrow \infty$. Now, we prove that $\widehat{u}$ solves (26). Taking limits in (40), we get

$$
\widehat{u}=\sum_{i=1}^{\infty} A_{i} \widehat{\Psi}_{i} \text {. }
$$

Note that

$$
\begin{aligned}
L \widehat{u} & =\sum_{i=1}^{\infty} A_{i} L \widehat{\Psi}_{i}, \\
(L \widehat{u})\left(x_{k}, t_{k}\right) & =\sum_{i=1}^{\infty} A_{i} L \widehat{\Psi}_{i}\left(x_{k}, t_{k}\right) \\
& =\sum_{i=1}^{\infty} A_{i}\left\langle L \widehat{\Psi}_{i}, G_{\left(x_{k}, t_{k}\right)}\right\rangle_{\widehat{W}}
\end{aligned}
$$

$$
\begin{aligned}
& =\sum_{i=1}^{\infty} A_{i}\left\langle L \widehat{\Psi}_{i}, \varphi_{k}\right\rangle_{\widehat{W}} \\
& =\sum_{i=1}^{\infty} A_{i}\left\langle\widehat{\Psi}_{i}, L^{*} \varphi_{k}\right\rangle_{W} \\
& =\sum_{i=1}^{\infty} A_{i}\left\langle\widehat{\Psi}_{i}, \Psi_{k}\right\rangle_{W} .
\end{aligned}
$$

In view of (47), we have

$$
\operatorname{Lu}\left(x_{l}, t_{l}\right)=M\left(x_{l}, t_{l}, u_{l-1}\left(x_{l}, t_{l}\right)\right) .
$$

Since $\left\{\left(x_{i}, t_{i}\right)\right\}_{i=1}^{\infty}$ is dense in $\Omega$, for each $(y, s) \in \Omega$, there exists a subsequence $\left\{\left(x_{n_{j}}, t_{n_{j}}\right)\right\}_{j=1}^{\infty}$ such that

$$
\left(x_{n_{j}}, t_{n_{j}}\right) \longrightarrow(y, s), \quad j \longrightarrow \infty .
$$

We know that

$$
\operatorname{Lu}\left(x_{n_{j}}, t_{n_{j}}\right)=M\left(x_{n_{j}}, t_{n_{j}}, u_{n_{j}-1}\left(x_{n_{j}}, t_{n_{j}}\right)\right) .
$$

Let $j \rightarrow \infty$. By the continuity of $f$, we have

$$
(L \widehat{u})(y, s)=M(y, s, \widehat{u}(y, s)),
$$

which indicates that $\widehat{u}$ satisfies (26).

Remark 19. In the same manner, it can be proved that

$$
\left\|\frac{\partial u_{n}}{\partial x}-\frac{\partial u}{\partial x}\right\| \longrightarrow 0, \quad n \longrightarrow \infty,
$$

where

$$
\frac{\partial u}{\partial x}=\sum_{i=1}^{\infty} A_{i} \frac{\partial \widehat{\Psi}_{i}}{\partial x}, \quad \frac{\partial u_{n}}{\partial x}=\sum_{i=1}^{n} A_{i} \frac{\partial \widehat{\Psi}_{i}}{\partial x},
$$

and $A_{i}$ is given by (47). 
TABLE 7: RMS errors for Example 21.

\begin{tabular}{lcccccc}
\hline & {$[10]$} & RKM & {$[10]$} & RKM & {$[10]$} & RKM \\
$N$ & $\alpha=20$ & $\alpha=20$ & $\alpha=20$ & $\alpha=20$ & $\alpha=10$ & $\beta=5$ \\
& $\beta=10$ & $\beta=10$ & $\beta=10$ & $t=10$ & $t=1.0$ & $t=0.5$ \\
\hline 5 & $t=0.5$ & $t=0.5$ & $3.4 \times 10^{-6}$ & $2.3 \times 10^{-8}$ & $3.6 \times 10^{-6}$ & $t=0.5$ \\
7 & $3.2 \times 10^{-7}$ & $2.8 \times 10^{-8}$ & $3.7 \times 10^{-9}$ & $5.9 \times 10^{-11}$ & $4.1 \times 10^{-10}$ & $9.6 \times 10^{-11}$ \\
9 & $2.0 \times 10^{-10}$ & $5.7 \times 10^{-7}$ & $2.4 \times 10^{-12}$ & $4.8 \times 10^{-13}$ & $3.5 \times 10^{-13}$ & $1.9 \times 10^{-12}$ \\
11 & $2.3 \times 10^{-13}$ & $2.7 \times 10^{-16}$ & $1.0 \times 10^{-15}$ & $2.3 \times 10^{-16}$ & $3.4 \times 10^{-16}$ & $1.3 \times 10^{-16}$ \\
\hline
\end{tabular}

TABLE 8: Numerical results for Example 22 for $t=0.5$.

\begin{tabular}{lccccccc}
\hline & $\mathrm{AE}$ & $\mathrm{RE}$ & & $\mathrm{AE}$ & $\mathrm{RE}$ & $\mathrm{AE}$ & $\mathrm{RE}$ \\
$x$ & $\alpha=20$ & $\alpha=20$ & $\mathrm{CPU}$ time $(\mathrm{s})$ & $\alpha=10$ & $\alpha=10$ & $\alpha=50$ & $\alpha=50$ \\
& $\beta=10$ & $\beta=10$ & & $\beta=5$ & $\beta=5$ & $\beta=2$ \\
\hline 0.9829 & $6.3 \times 10^{-8}$ & $1.9 \times 10^{-7}$ & 2.231 & $1.8 \times 10^{-5}$ & $5.8 \times 10^{-5}$ & $6.5 \times 10^{-6}$ & $2.0 \times 10^{-5}$ \\
0.9903 & $7.2 \times 10^{-7}$ & $1.1 \times 10^{-6}$ & 2.540 & $4.5 \times 10^{-4}$ & $7.2 \times 10^{-4}$ & $7.5 \times 10^{-4}$ & $1.1 \times 10^{-3}$ \\
0.9938 & $4.9 \times 10^{-8}$ & $1.7 \times 10^{-7}$ & 2.230 & $3.6 \times 10^{-5}$ & $1.2 \times 10^{-4}$ & $8.9 \times 10^{-6}$ & $3.2 \times 10^{-5}$ \\
0.9957 & $6.4 \times 10^{-8}$ & $9.6 \times 10^{-8}$ & 2.680 & $2.5 \times 10^{-6}$ & $3.8 \times 10^{-6}$ & $1.5 \times 10^{-4}$ & $2.3 \times 10^{-4}$ \\
\hline
\end{tabular}

\section{Numerical Results}

To test the accuracy of the present method, some numerical experiments are presented in this section. Using our method, we chose 36 points in $\Omega$ and obtained the approximate solution $u_{36}$. The comparison between interpolating scaling function method [11] and RKM for different values of $\alpha, \beta$, and $t$ is given in Tables 4 and 5 . We solve these examples for a set of points

$$
\left\{x_{1}=0, \ldots, x_{i}=(i-1) h, \ldots, x_{N}=1\right\}, \quad h=\frac{1}{N-1} \text {. }
$$

In Tables 7 and 10 we calculate the RMS error by the following formula:

$$
\text { RMS error }=\sqrt{\frac{\sum_{i=1}^{N+1}\left(u-u_{36}\right)^{2}}{N+1}} .
$$

It can be seen from Tables 4 and 5 and 7-10 that the results obtained by the RKM are more accurate than those obtained by the methods in $[10,11]$. This indicates that RKM is a reliable method. The CPU time $(s)$ is given in Tables $1,2,3,4,5,6,7,8$, 9 , and 10. Numerical solutions are described in the extended domain $[-4,4] \times[-3,3]$. The comparison of RMS error is given for our method and Chebyshev method.

Example 20. Consider the following telegraph equation with initial and boundary conditions:

$$
\begin{gathered}
\frac{\partial^{2} u}{\partial t^{2}}(x, t)+2 \alpha \frac{\partial u}{\partial t}(x, t)+\beta^{2} u(x, t)=\frac{\partial^{2} u}{\partial x^{2}}+f(x, t) \\
u(x, 0)=\varphi_{1}(x)=\sinh (x) \\
\frac{\partial u}{\partial t}(x, 0)=\varphi_{2}(x)=-2 \sinh (x)
\end{gathered}
$$

$$
\begin{gathered}
u(0, t)=g_{0}(t)=0, \quad t \geq 0, \\
u(1, t)=g_{1}(t)=\exp (-2 t) \sinh (1), \quad t \geq 0,
\end{gathered}
$$

where

$$
f(x, t)=\left(3-4 \alpha+\beta^{2}\right) \exp (-2 t) \sinh (x) .
$$

The exact solution of (64) is given by [11]

$$
u(x, t)=\exp (-2 t) \sinh (x) .
$$

If we apply

$$
\begin{aligned}
v(x, t)= & u(x, t)-x \sinh (1)(\exp (-2 t)-1+2 t) \\
& +\sinh (x)(2 t-1)
\end{aligned}
$$

to (64), then the following equation (68) is obtained:

$$
\begin{aligned}
& \frac{\partial^{2} v}{\partial t^{2}}-\frac{\partial^{2} v}{\partial x^{2}}+2 \alpha \frac{\partial v}{\partial t}+\beta^{2} v(x, t)=M(x, t), \\
& v(x, 0)=\frac{\partial v}{\partial t}(x, 0)=v(0, t)=v(1, t)=0,
\end{aligned}
$$

where

$$
\begin{aligned}
M(x, t)= & \left(3-4 \alpha+\beta^{2}\right) \exp (-2 t) \sinh (x) \\
& -\sinh (x)(2 t-1) \\
& -2 \alpha \sinh (1)(-2 \exp (-2 t)+2) x \\
& +4 \alpha \sinh (x) \\
& -\beta^{2} \sinh (1)(\exp (-2 t)-1+2 t) x \\
& +\beta^{2} \sinh (x)(2 t-1)-4 x \exp (-2 t) \sinh (1)
\end{aligned}
$$


TABLE 9: Numerical results for Example 22 for $t=1.0$.

\begin{tabular}{|c|c|c|c|c|c|c|c|}
\hline$x$ & CPU time (s) & $\begin{array}{c}\mathrm{AE} \\
\alpha=20 \\
\beta=10\end{array}$ & $\begin{array}{c}\mathrm{RE} \\
\alpha=20 \\
\beta=10\end{array}$ & $\begin{array}{c}\mathrm{AE} \\
\alpha=10 \\
\beta=5\end{array}$ & $\begin{array}{c}\mathrm{RE} \\
\alpha=10 \\
\beta=5\end{array}$ & $\begin{array}{c}\mathrm{AE} \\
\alpha=50 \\
\beta=2\end{array}$ & $\begin{array}{c}\mathrm{RE} \\
\alpha=50 \\
\beta=2\end{array}$ \\
\hline 0.9829 & 2.587 & $2.7 \times 10^{-7}$ & $4.3 \times 10^{-7}$ & $3.2 \times 10^{-5}$ & $5.1 \times 10^{-5}$ & $6.9 \times 10^{-5}$ & $1.1 \times 10^{-4}$ \\
\hline 0.9903 & 2.058 & $9.2 \times 10^{-7}$ & $8.7 \times 10^{-7}$ & $1.0 \times 10^{-5}$ & $9.6 \times 10^{-6}$ & $1.0 \times 10^{-4}$ & $9.6 \times 10^{-5}$ \\
\hline 0.9938 & 2.262 & $2.5 \times 10^{-5}$ & $4.4 \times 10^{-5}$ & $6.9 \times 10^{-5}$ & $1.2 \times 10^{-4}$ & $1.1 \times 10^{-4}$ & $1.9 \times 10^{-4}$ \\
\hline 0.9957 & 2.246 & $1.7 \times 10^{-4}$ & $1.6 \times 10^{-4}$ & $6.9 \times 10^{-4}$ & $6.3 \times 10^{-4}$ & $1.4 \times 10^{-4}$ & $1.3 \times 10^{-4}$ \\
\hline
\end{tabular}

TABLE 10: RMS errors for Example 22.

\begin{tabular}{|c|c|c|c|c|c|c|}
\hline \multirow{4}{*}{$N$} & [10] & RKM & [10] & RKM & {$[10]$} & RKM \\
\hline & $\alpha=10$ & $\alpha=10$ & $\alpha=50$ & $\alpha=50$ & $\alpha=50$ & $\alpha=50$ \\
\hline & $\beta=5$ & $\beta=5$ & $\beta=2$ & $\beta=2$ & $\beta=2$ & $\beta=2$ \\
\hline & $t=1.0$ & $t=1.0$ & $t=0.5$ & $t=0.5$ & $t=1.0$ & $t=1.0$ \\
\hline 5 & $2.8 \times 10^{-5}$ & $9.8 \times 10^{-6}$ & $3.1 \times 10^{-5}$ & $2.3 \times 10^{-6}$ & $6.9 \times 10^{-4}$ & $1.1 \times 10^{-5}$ \\
\hline 7 & $1.3 \times 10^{-5}$ & $6.7 \times 10^{-7}$ & $1.5 \times 10^{-6}$ & $5.9 \times 10^{-7}$ & $2.2 \times 10^{-5}$ & $9.6 \times 10^{-6}$ \\
\hline 9 & $2.8 \times 10^{-7}$ & $7.8 \times 10^{-8}$ & $1.8 \times 10^{-8}$ & $4.8 \times 10^{-10}$ & $6.3 \times 10^{-7}$ & $1.9 \times 10^{-9}$ \\
\hline 11 & $6.1 \times 10^{-9}$ & $5.3 \times 10^{-11}$ & $7.8 \times 10^{-10}$ & $2.3 \times 10^{-11}$ & $1.6 \times 10^{-8}$ & $1.3 \times 10^{-10}$ \\
\hline
\end{tabular}

After homogenizing the initial and boundary conditions and using the above method, we obtain Tables 1-4 and Figure 1.

Example 21. Consider the following telegraph equation with initial and boundary conditions:

$$
\begin{gathered}
\frac{\partial^{2} u}{\partial t^{2}}+2 \alpha \frac{\partial u}{\partial t}+\beta^{2} u(x, t)=\frac{\partial^{2} u}{\partial x^{2}}+f(x, t), \\
u(x, 0)=\varphi_{1}(x)=\sin (x), \\
\frac{\partial u}{\partial t}(x, 0)=\varphi_{2}(x)=0 \\
u(0, t)=g_{0}(t)=0, \quad t \geq 0, \\
u(1, t)=g_{1}(t)=\cos (t) \sin (1), \quad t \geq 0,
\end{gathered}
$$

where

$$
f(x, t)=-2 \alpha \sin (t) \sin (x)+\beta^{2} \cos (t) \sin (x) .
$$

The exact solution of (70) is given by [11]

$$
u(x, t)=\cos (t) \sin (x) \text {. }
$$

If we apply

$$
v(x, t)=u(x, t)-x \sin (1)(\cos (t)-1)-\sin (x)
$$

to $(70)$, then the following $(74)$ is obtained:

$$
\begin{gathered}
\frac{\partial^{2} v}{\partial t^{2}}(x, t)-\frac{\partial^{2} v}{\partial x^{2}}(x, t)+2 \alpha \frac{\partial v}{\partial t} \\
+\beta^{2} v(x, t)=M(x, t), \\
v(x, 0)=\frac{\partial v}{\partial t}(x, 0)=v(0, t)=v(1, t)=0,
\end{gathered}
$$

where

$$
\begin{aligned}
M(x, t)= & -2 \alpha \sin (t) \sin (x)+\beta^{2} \cos (t) \sin (x)-\sin (x) \\
& -2 \alpha \sin (t) \sinh (1) x+x \cos (t) \sin (1) \\
& -\beta^{2} x \sin (1)(\cos (t)-1)-\beta^{2} \sin (x)
\end{aligned}
$$

After homogenizing the initial and boundary conditions and using the above method, we obtain Tables 5-7 and Figures 2 and 3 .

Example 22. Consider the following telegraph equation with initial and boundary conditions:

$$
\begin{gathered}
\frac{\partial^{2} u}{\partial t^{2}}+2 \alpha \frac{\partial u}{\partial t}+\beta^{2} u(x, t)=\frac{\partial^{2} u}{\partial x^{2}}+f(x, t), \\
u(x, 0)=\varphi_{1}(x)=\tan \left(\frac{x}{2}\right), \\
\frac{\partial u}{\partial t}(x, 0)=\varphi_{2}(x)=\frac{\left(1+\tan ^{2}(x / 2)\right)}{2}, \\
u(0, t)=g_{0}(t)=\tan \left(\frac{t}{2}\right), \quad t \geq 0, \\
u(1, t)=g_{1}(t)=\tan \left(\frac{1+t}{2}\right), \quad t \geq 0,
\end{gathered}
$$

where

$$
f(x, t)=\alpha\left(1+\tan ^{2}\left(\frac{x+t}{2}\right)\right)+\beta^{2} \tan \left(\frac{x+t}{2}\right) .
$$

The exact solution of (76) is given by [10]

$$
u(x, t)=\tan \left(\frac{x+t}{2}\right) .
$$




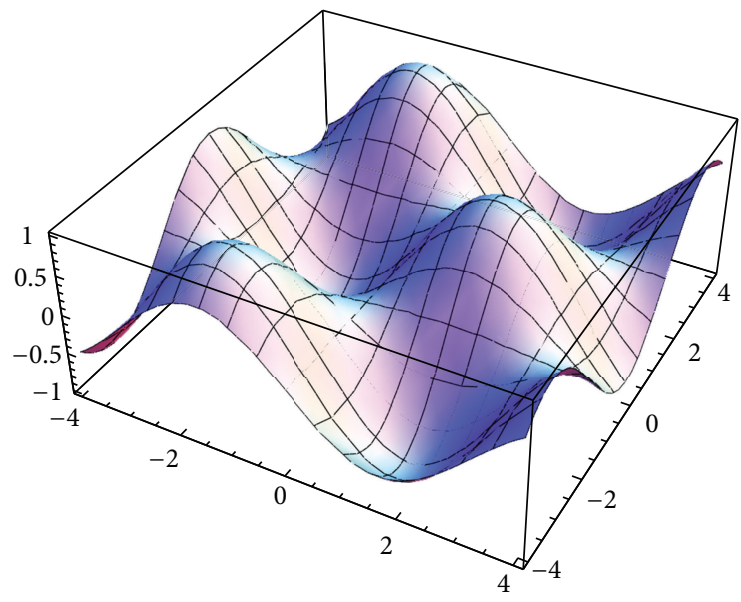

(a)

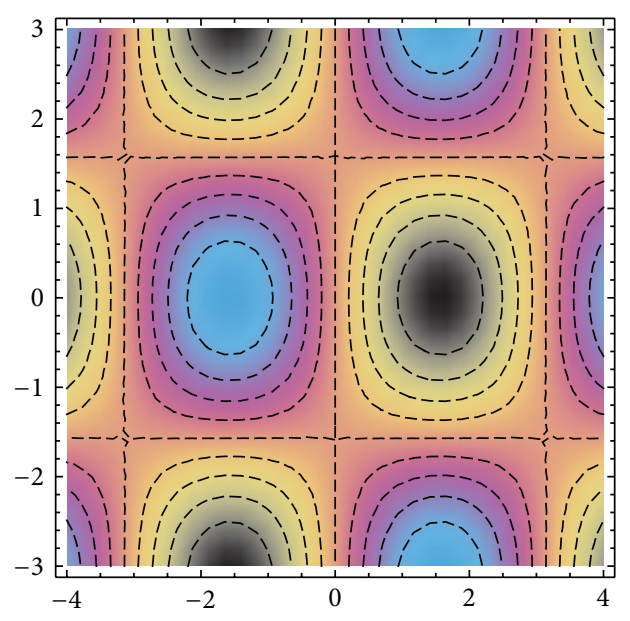

(b)

Figure 3: Plots of numerical results for Example $21(\alpha=6, \beta=2)$.

If we apply

$$
\begin{aligned}
v(x, t)= & u(x, t)+(x-1) \tan \left(\frac{t}{2}\right) \\
& -x \tan \left(\frac{1+t}{2}\right)-\tan \left(\frac{x}{2}\right) \\
& \times\left(1+\left(\frac{t \tan (x / 2)}{2}\right)\right) \\
& +x \tan \left(\frac{1}{2}\right)\left(1+\left(\frac{t \tan (1 / 2)}{2}\right)\right)
\end{aligned}
$$

$$
v(x, 0)=\frac{\partial v}{\partial t}(x, 0)=v(0, t)=v(1, t)=0,
$$

where

$$
\begin{aligned}
M(x, t)= & \alpha\left(1+\tan ^{2}\left(\frac{x+t}{2}\right)\right)+\beta^{2} \tan \left(\frac{x+t}{2}\right) \\
& +\left(\frac{\tan (x / 2)}{2}+\frac{t}{4}+\frac{3 t}{4} \tan ^{2}\left(\frac{x}{2}\right)\right) \\
& \times\left(1+\tan ^{2}\left(\frac{x}{2}\right)\right) \\
& +(x-1) \alpha\left(1+\tan ^{2}\left(\frac{t}{2}\right)\right) \\
& -\alpha x\left(1+\tan ^{2}\left(\frac{t+1}{2}\right)\right)-\alpha \tan \left(\frac{x}{2}\right) \\
& +\alpha x \tan ^{2}\left(\frac{1}{2}\right)+\beta^{2}(x-1) \tan \left(\frac{t}{2}\right) \\
& -\beta^{2} x \tan ^{2}\left(\frac{1+t}{2}\right)-\beta^{2} \tan \left(\frac{x}{2}\right)
\end{aligned}
$$

$$
\begin{aligned}
& \times\left(1+\frac{t \tan (x / 2)}{2}\right) \\
& +\beta^{2} x \tan \left(\frac{1}{2}\right)\left(1+\frac{t \tan (1 / 2)}{2}\right) \\
& \times \frac{x-1}{2} \tan \left(\frac{t}{2}\right)\left(1+\tan ^{2}\left(\frac{t}{2}\right)\right) \\
& -\frac{x}{2} \tan \left(\frac{1+t}{2}\right)\left(1+\tan ^{2}\left(\frac{1+t}{2}\right)\right) .
\end{aligned}
$$

After homogenizing the initial and boundary conditions and using the above method, we obtain Tables 8-10 and Figure 4.

Remark 23. In Tables 1-9, we abbreviate the exact solution and the approximate solution by AS and ES, respectively. AE stands for the absolute error, that is, the absolute value of the difference of the exact solution and the approximate solution, while RE indicates the relative error, that is, the absolute error divided by the absolute value of the exact solution.

\section{Conclusion}

In this study, a second-order one-dimensional telegraph equation with initial and boundary conditions was solved by reproducing kernel Hilbert space method. We described the method and used it in some test examples in order to show its applicability and validity in comparison with exact and other numerical solutions. The obtained results show that this approach can solve the problem effectively and need few computations. The satisfactory results that we obtained were compared with the results that were obtained by $[10,11]$. Numerical experiments on test examples show that our proposed schemes are of high accuracy and support the theoretical results. As shown in Tables 7 and 10 our results are better than the results that were obtained by [10]. According to these results, it is possible to apply RKM to linear and nonlinear differential equations with initial and 


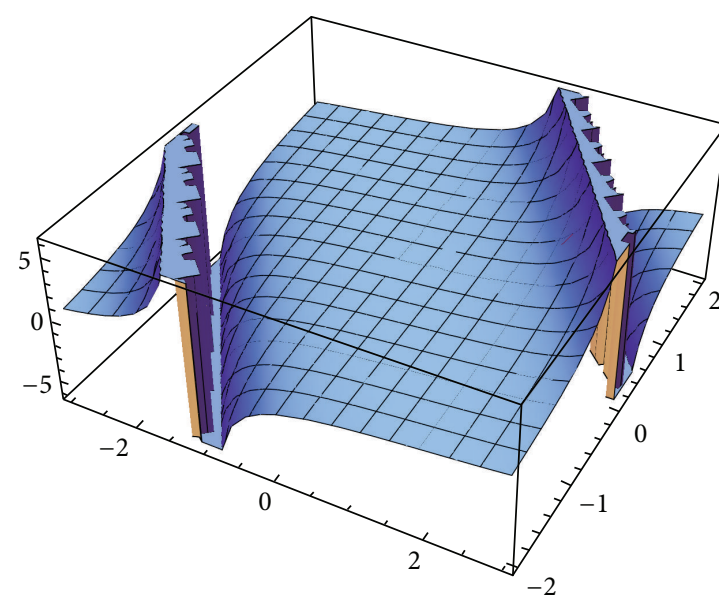

(a)

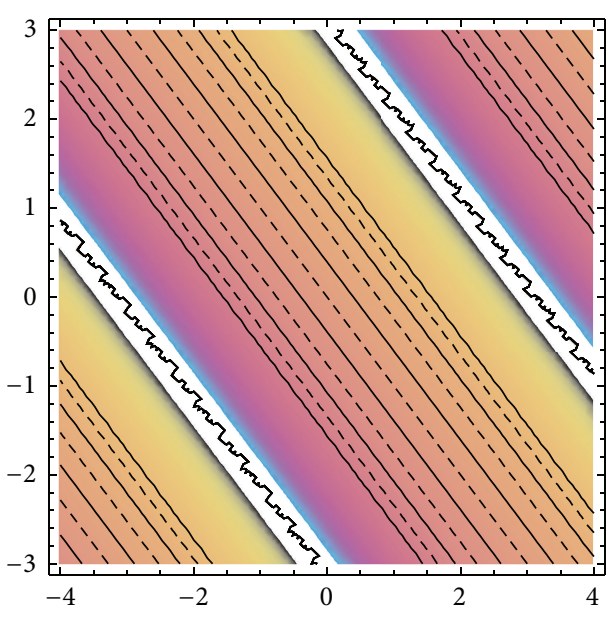

(b)

FIGURE 4: Plots of numerical results for Example $22(\alpha=10, \beta=5)$.

boundary conditions. It has been shown that the obtained results are uniformly convergent and the operator that was used is a bounded linear operator. From the results, RKM can be applied to high dimensional partial differential equations, integral equations, and fractional differential equations without any transformation or discretization, and good results can be obtained.

\section{References}

[1] M. Dehghan, "On the solution of an initial-boundary value problem that combines Neumann and integral condition for the wave equation," Numerical Methods for Partial Differential Equations, vol. 21, no. 1, pp. 24-40, 2005.

[2] R. K. Mohanty, M. K. Jain, and K. George, "On the use of high order difference methods for the system of one space second order nonlinear hyperbolic equations with variable coefficients," Journal of Computational and Applied Mathematics, vol. 72, no. 2, pp. 421-431, 1996.

[3] E. H. Twizell, "An explicit difference method for the wave equation with extended stability range," BIT, vol. 19, no. 3, pp. 378-383, 1979.

[4] R. K. Mohanty, "An unconditionally stable difference scheme for the one-space-dimensional linear hyperbolic equation," Applied Mathematics Letters, vol. 17, no. 1, pp. 101-105, 2004.

[5] A. Mohebbi and M. Dehghan, "High order compact solution of the one-space-dimensional linear hyperbolic equation," Numerical Methods for Partial Differential Equations, vol. 24, no. 5, pp. 1222-1235, 2008.

[6] M. Dehghan, "Finite difference procedures for solving a problem arising in modeling and design of certain optoelectronic devices," Mathematics and Computers in Simulation, vol. 71, no. 1, pp. 16-30, 2006.

[7] M. Dehghan and A. Shokri, "A numerical method for solving the hyperbolic telegraph equation," Numerical Methods for Partial Differential Equations, vol. 24, no. 4, pp. 1080-1093, 2008.

[8] H. Yao, "Reproducing kernel method for the solution of nonlinear hyperbolic telegraph equation with an integral condition," Numerical Methods for Partial Differential Equations, vol. 27, no. 4, pp. 867-886, 2011.
[9] S. A. Yousefi, "Legendre multiwavelet Galerkin method for solving the hyperbolic telegraph equation," Numerical Methods for Partial Differential Equations, vol. 26, no. 3, pp. 535-543, 2010.

[10] M. Dehghan and M. Lakestani, “The use of Chebyshev cardinal functions for solution of the second-order one-dimensional telegraph equation," Numerical Methods for Partial Differential Equations, vol. 25, no. 4, pp. 931-938, 2009.

[11] M. Lakestani and B. N. Saray, "Numerical solution of telegraph equation using interpolating scaling functions," Computers \& Mathematics with Applications, vol. 60, no. 7, pp. 1964-1972, 2010.

[12] M. Dehghan and A. Ghesmati, "Solution of the second-order one-dimensional hyperbolic telegraph equation by using the dual reciprocity boundary integral equation (DRBIE) method," Engineering Analysis with Boundary Elements, vol. 34, no. 1, pp. 51-59, 2010.

[13] A. N. Tikhonov and A. A. Samarskiü, Equations of Mathematical Physics, Dover, New York, NY, USA, 1990.

[14] N. Aronszajn, “Theory of reproducing kernels," Transactions of the American Mathematical Society, vol. 68, pp. 337-404, 1950.

[15] M. Inc, A. Akgül, and A. Kılıçman, "Explicit solution of telegraph equation based on reproducing kernel method," Journal of Function Spaces and Applications, vol. 2012, p. 23, 2012.

[16] M. Inc and A. Akgül, "The reproducing kernel Hilbert space method for solving Troesch's problem," Journal of the Association of Arab Universities for Basic and Applied Sciences. In press.

[17] M. Inc, A. Akgül, and A. Kılıçman, "A new application of the reproducing kernel Hilbert space method to solve MHD JefferyHamel flows problem in nonparallel walls," Abstract and Applied Analysis, vol. 2013, Article ID 239454, 12 pages, 2013.

[18] M. Inc, A. Akgül, and F. Geng, "Reproducing kernel Hilbert space method for solving Bratu's problem," Bulletin of the Malaysian Mathematical Sciences Society. In press.

[19] M. Inc, A. Akgül, and A. Kılıçman, "On solving KdV equation using reproducing kernel Hilbert space method," Abstract and Applied Analysis, vol. 2013, Article ID 578942, 11 pages, 2013.

[20] W. Jiang and Y. Lin, "Representation of exact solution for the time-fractional telegraph equation in the reproducing kernel 
space," Communications in Nonlinear Science and Numerical Simulation, vol. 16, no. 9, pp. 3639-3645, 2011.

[21] Y. Wang, L. Su, X. Cao, and X. Li, "Using reproducing kernel for solving a class of singularly perturbed problems," Computers \& Mathematics with Applications, vol. 61, no. 2, pp. 421-430, 2011.

[22] F. Geng and M. Cui, "A novel method for nonlinear two-point boundary value problems: combination of ADM and RKM," Applied Mathematics and Computation, vol. 217, no. 9, pp. 46764681, 2011.

[23] F. Geng and F. Shen, "Solving a Volterra integral equation with weakly singular kernel in the reproducing kernel space," Mathematical Sciences Quarterly Journal, vol. 4, no. 2, pp. 159$170,2010$.

[24] F. Geng and M. Cui, "Homotopy perturbation-reproducing kernel method for nonlinear systems of second order boundary value problems," Journal of Computational and Applied Mathematics, vol. 235, no. 8, pp. 2405-2411, 2011.

[25] M. Cui and Y. Lin, Nonlinear Numerical Analysis in the Reproducing Kernel Space, Nova Science, New York, NY, USA, 2009.

[26] A. M. Krall, Hilbert Space, Boundary Value Problems and Orthogonal Polynomials, vol. 133 of Operator Theory: Advances and Applications, Birkhäuser, Basel, Switzerland, 2002. 


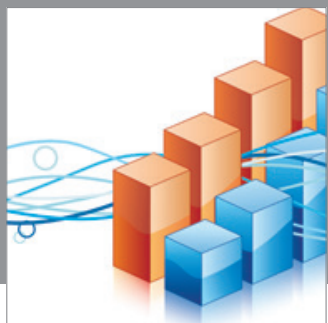

Advances in

Operations Research

mansans

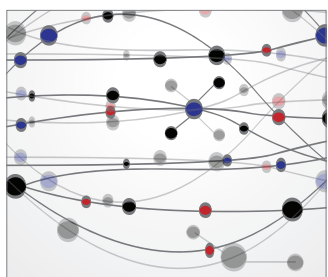

The Scientific World Journal
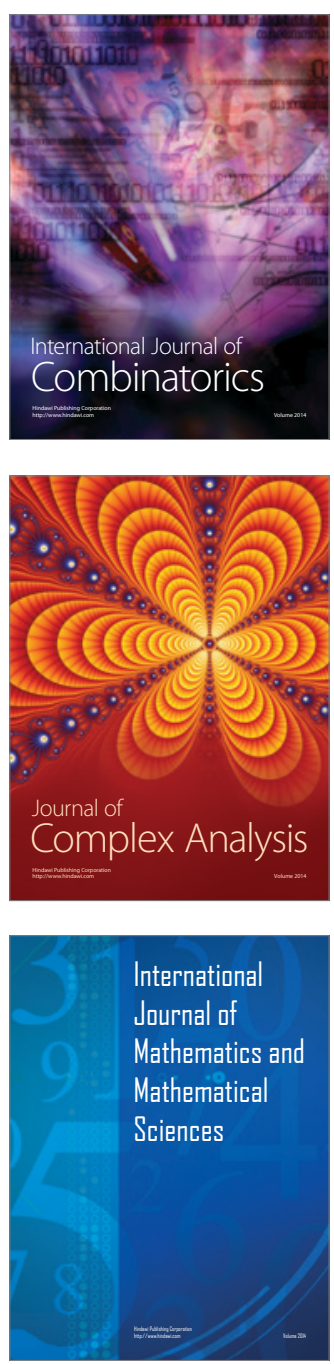
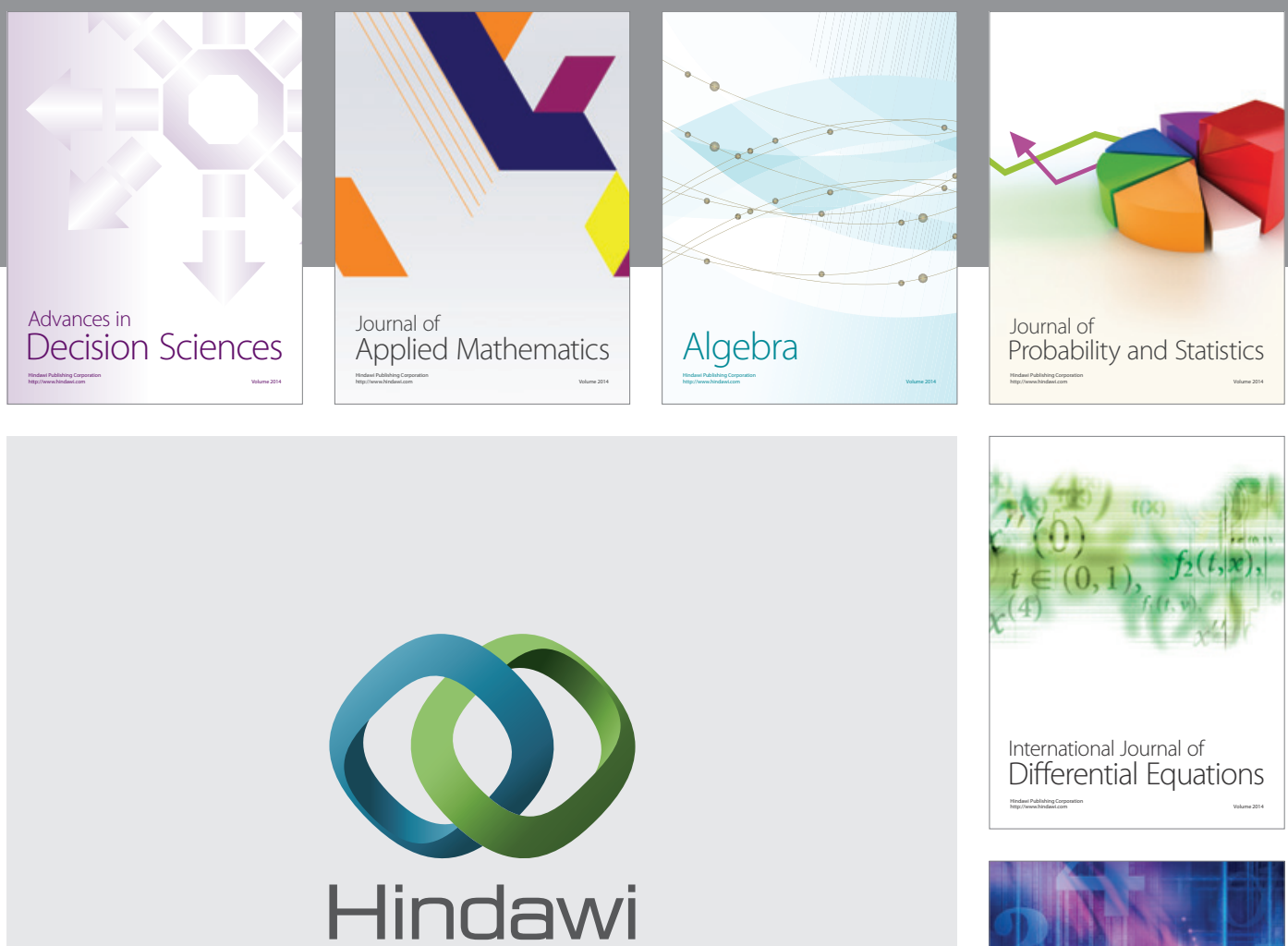

Submit your manuscripts at http://www.hindawi.com
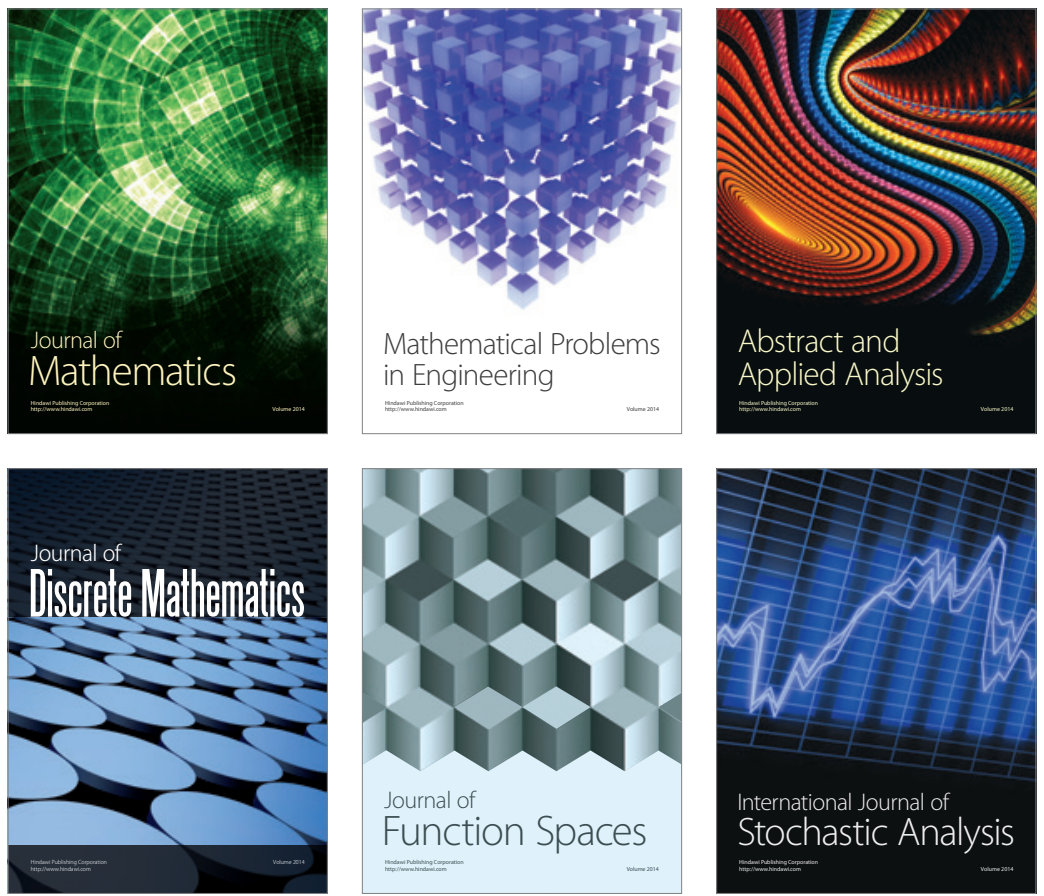

Journal of

Function Spaces

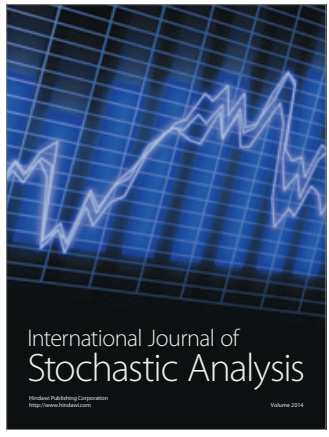

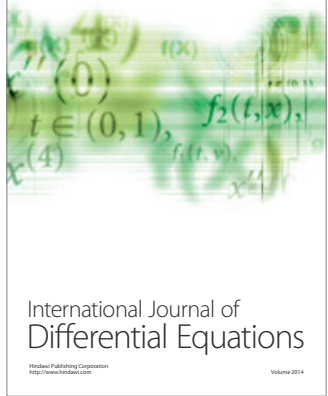
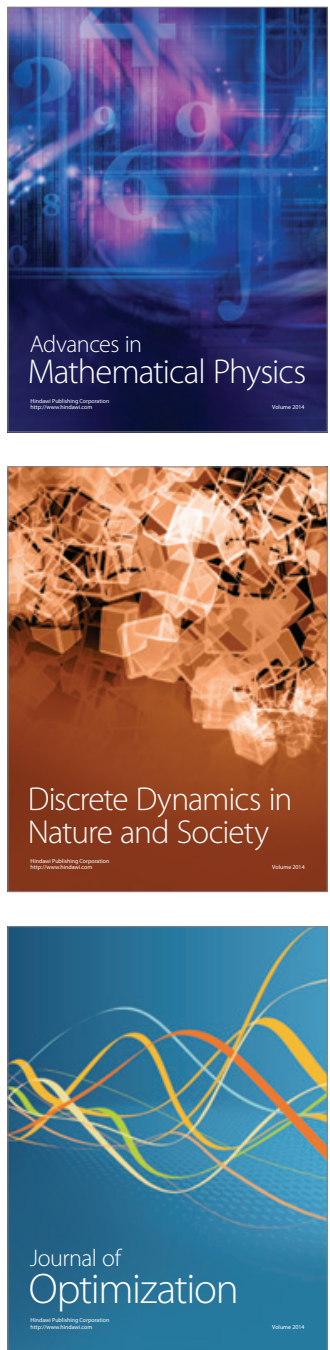